\title{
The neuro-pathophysiology of temporomandibular disorders-related pain: a systematic review of structural and functional MRI studies
}

Yuanyuan Yin ${ }^{1,2+}$, Shushu He ${ }^{2+}$, Jingchen $\mathrm{Xu}^{2}$, Wanfang You ${ }^{1,3}$, Qian Li $\mathrm{i}^{1,3}$, Jingyi Long ${ }^{1,3}$, Lekai Luo ${ }^{1,3}$, Graham J. Kemp ${ }^{4}$, John A. Sweeney ${ }^{1,5}$, Fei Li ${ }^{1,3^{*}}$, Song Chen ${ }^{2^{*}}$ (i) and Qiyong Gong ${ }^{1,3}$

\begin{abstract}
Chronic pain surrounding the temporomandibular joints and masticatory muscles is often the primary chief complaint of patients with temporomandibular disorders (TMD) seeking treatment. Yet, the neuro-pathophysiological basis underlying it remains to be clarified. Neuroimaging techniques have provided a deeper understanding of what happens to brain structure and function in TMD patients with chronic pain. Therefore, we performed a systematic review of magnetic resonance imaging (MRI) studies investigating structural and functional brain alterations in TMD patients to further unravel the neurobiological underpinnings of TMD-related pain. Online databases (PubMed, EMBASE, and Web of Science) were searched up to August 3, 2019, as complemented by a hand search in reference lists. A total of 622 papers were initially identified after duplicates removed and 25 studies met inclusion criteria for this review. Notably, the variations of MRI techniques used and study design among included studies preclude a meta-analysis and we discussed the findings qualitatively according to the specific neural system or network the brain regions were involved in. Brain changes were found in pathways responsible for abnormal pain perception, including the classic trigemino-thalamo-cortical system and the lateral and medial pain systems. Dysfunction and maladaptive changes were also identified in the default mode network, the top-down antinociceptive periaqueductal gray-raphe magnus pathway, as well as the motor system. TMD patients displayed altered brain activations in response to both innocuous and painful stimuli compared with healthy controls. Additionally, evidence indicates that splint therapy can alleviate TMD-related symptoms by inducing functional brain changes. In summary, MRI research provides important novel insights into the altered neural manifestations underlying chronic pain in TMD.
\end{abstract}

Keywords: Temporomandibular disorders, Chronic pain, Magnetic resonance imaging, Gray matter, White matter, Brain structure and function, Splint therapy Psychoradiology

\footnotetext{
* Correspondence: charlie_lee@qq.com; songchen882002@hotmail.com

${ }^{\dagger}$ Yuanyuan Yin and Shushu He contributed equally to this work.

${ }^{1}$ Huaxi MR Research Center (HMRRC), Department of Radiology, West China

Hospital of Sichuan University, Chengdu 610041, Sichuan, People's Republic of China

${ }^{2}$ State Key Laboratory of Oral Diseases, National Clinical Research Center for

Oral Diseases, Department of Orthodontics, West China School of

Stomatology, Sichuan University, Chengdu 610041, Sichuan, People's

Republic of China

Full list of author information is available at the end of the article
}

(c) The Author(s). 2020 Open Access This article is licensed under a Creative Commons Attribution 4.0 International License, which permits use, sharing, adaptation, distribution and reproduction in any medium or format, as long as you give appropriate credit to the original author(s) and the source, provide a link to the Creative Commons licence, and indicate if changes were made. The images or other third party material in this article are included in the article's Creative Commons licence, unless indicated otherwise in a credit line to the material. If material is not included in the article's Creative Commons licence and your intended use is not permitted by statutory regulation or exceeds the permitted use, you will need to obtain permission directly from the copyright holder. To view a copy of this licence, visit http://creativecommons.org/licenses/by/4.0/ The Creative Commons Public Domain Dedication waiver (http://creativecommons.org/publicdomain/zero/1.0/) applies to the data made available in this article, unless otherwise stated in a credit line to the data. 


\section{Introduction}

Temporomandibular disorders (TMD) are a subgroup of craniofacial pain disorders involving pain and dysfunction of the temporomandibular joint (TMJ), masticatory muscles and associated musculoskeletal structures of the head and neck. It is the commonest cause of nondental pain in the orofacial region [1]. Patients with TMD frequently suffer from localized pain, impaired jaw movement and noise from TMJ during jaw movement, as well as less specific symptoms including ear pain and stuffiness, tinnitus, dizziness, neck pain and headache $[2,3]$. Although not life-threatening, it can affect oral healthrelated quality of life, and the symptoms can be chronic and difficult to manage $[4,5]$. However, as with many chronic pain syndromes, the neurobiological mechanisms pertaining to pain in TMD remain to be clarified.

Initially, TMD-related chronic pain was considered to be caused primarily by peripheral factors, such as chronic inflammation of the TMJ, microtrauma of the masticatory muscles, and oromotor dysfunction. However, the correlation between pain severity and tissue pathology is often poor, and not all patients have clearly identifiable peripheral etiological factors [6-8]. Moreover, TMD patients may have pain in other body areas $[9,10]$, and there is high comorbidity with functional syndromes such as fibromyalgia [11] and irritable bowel syndrome [12]. Such evidence implicates central nervous system dysfunction in pain associated with TMD [13-15].

Previous studies have thoroughly investigated changes in brain activity when experiencing clinical pain [16]. Persistent nociceptive input to brain can induce maladaptive anatomical and physiological changes in the brain via pathology or compensation $[17,18]$. Functional and structural magnetic resonance imaging (MRI) methods have been widely used separately or combined to explore brain alterations in patients with chronic pain [19-21], including TMD, for better understanding of the neural mechanisms of pain perception and chronification. MRI studies in TMD have provided evidence for structural and functional changes within the ascending trigemino-thalamo-cortical pathway involving the trigeminal nerve root, spinal tract subnucleus caudalis $(\mathrm{SpVc})$, thalamus, and primary somatosensory cortex (S1). For example, SpVc, which lies within the caudal brainstem and processes orofacial nociceptive input from the trigeminal peripheral nerve ending, shows decreased gray matter volume (GMV) [22] and elevated cerebral blood flow (CBF) [23] in TMD patients. Brain alterations have also been found in widespread brain regions involved in the lateral and medial pain systems, the default mode network (DMN) and the top-down antinociceptive periaqueductal gray (PAG)-raphe magnus pathway functioning for pain perception and modulation. These new findings of structural and functional alterations at network levels may either account for the pathogenesis or be the consequences of pain in TMD. Although there are systematic reviews and metaanalysis on brain alterations of patients with orofacial pain disorders within which TMD was involved [24-26], there has so far been no comprehensive review specifically focusing on TMD to highlight progress in this area of work.

Therefore, this systematic review was conducted to provide an overview of neuroimaging MRI studies that shed light on neuro-pathophysiological basis underlying TMD-related pain by defining associated structural and functional brain alterations. Studies reporting brain changes after splint treatment that have potential implications for clarifying the therapeutic mechanism of TMD were also reviewed. Finally, while reviewing progress in this field, consideration was also given to describing the limitations of previous work and suggesting future directions for neuroimaging investigations of TMD.

\section{Methods}

\section{Search strategy and study selection}

We followed the Preferred Reporting Items for Systematic reviews and Meta-Analyses (PRISMA) guidelines for data collection [27]. Studies were identified by searching electronic databases including PubMed, EMBASE, and Web of Science up to August 3, 2019. The following search terms were used: (TMD OR temporomandibular disorders) AND pain AND (neuroimaging OR (sMRI OR structural MRI) OR (DTI OR diffusion tensor imaging) OR (fMRI OR functional MRI) OR (ASL OR arterial spin labeling) OR (MRS OR magnetic resonance spectroscopy)). Additional publications were identified by manual search in reference lists. Studies were included according to the following criteria: 1) original publications in English from peer-reviewed journals; 2) studies conducted in patients with TMD diagnosed with Research Diagnostic Criteria for TMD (RDC/TMD) [28] or Diagnostic Criteria for TMD (DC/TMD) [29]; and 3) MRI studies exploring the brain structure and function of TMD patients. Exclusion criteria included: 1) conference abstracts, theoretical papers, and reviews; and 2) studies using magnetoencephalography or electroencephalography. Two of us (Y.Y.Y. and S.S.H.) independently conducted the literature search. The results of these two searches were compared and any inconsistencies were discussed, and a consensus decision reached about inclusion.

\section{Quality assessment}

A customized 9-point checklist based on the Strengthening the Reporting of Observational Studies in 
Epidemiology statement [30] was used for study quality and risk of bias assessment (Additional file 1). The checklist focused on characteristics of participants for considerations of the heterogeneity of diagnosis and the impact of medication and comorbidity on results. We also incorporated items assessing the imaging methodology and statistical analysis. Any study scoring $\geq 5$ was included in this systematic review. Two independent reviewers (Y.Y.Y. and S.S.H.) performed the assessment and disagreements were resolved by discussion to reach a consensus.

\section{Data analysis}

The present results of brain structural and functional differences between patients with TMD and controls were qualitatively described according to the specific brain system or network the brain regions were involved in. Altered brain activations in response to mechanical stimuli, the effects on the brain of splint treatment, as well as the association between brain alterations and other pain-related measurements were summarized and presented descriptively.

\section{Results}

\section{Search results and study characteristics}

Figure 1 shows a flowchart of the search and selection process. A total of 25 studies published between January 1, 2010 and August 3, 2019 were included (details in Tables 1 and 2 and Additional file 2). Twenty-four MRI studies [22, 23, 31, 32, 35, 39, 41-43, 45-59] compared patients with TMD and healthy controls, while 1 longitudinal study recruited only patients [60].

\section{Neuroimaging methods employed in the reviewed studies}

Of the 25 studies, 4 studies [31, 32, 39, 41] used the single modality of structural MRI (sMRI) to assess GMV changes by voxel-based morphology and/or cortical thickness alterations using surface-based morphometry; of these, 2 studies [31, 32] performed a whole-brain analysis and 2 studies $[39,41]$ used a region of interest (ROI) approach. Three studies [22, 43, 45] combined sMRI and diffusion tensor imaging (DTI) to explore both GMV and white matter microstructure. One study [42] employed the single modality of DTI to investigate changes of fractional anisotropy (FA) and mean diffusivity (MD) in patients with TMD. Four studies [46-49] used resting-state fMRI (rs-fMRI) to evaluate spontaneous neural function and functional connectivity (FC). Nine studies [50-57, 60] used task-state fMRI (ts-fMRI) to investigate regional changes in neural activity during pain perception and modulation, of which 1 study [51] combined resting and task state fMRI and 1 study [50] mixed 3 modalities of ts-fMRI, DTI, and arterial spin labeling (ASL). One study [23] used the single modality of
ASL to measure CBF in the whole brain and the brainstem in two steps. Three studies $[35,58,59]$ used proton magnetic resonance spectroscopy $\left({ }^{1} \mathrm{H}\right.$ MRS) to identify the metabolites in the brain of TMD patients; of these 2 studies $[58,59]$ focused on the posterior insular and 1 study [35] used both sMRI and ${ }^{1} \mathrm{H}$ MRS.

\section{Characteristics of the subject populations}

Notably, there was some overlap in the patient cohorts. Six studies from the University of Toronto recruited the same subjects (17 TMD patients and 17 healthy controls) [39, 41-43, 46, 54]. The patients in 3 research groups of studies partially overlapped: first, 5 studies from the University of Sydney [22, 23, 35, 45, 50]; second, 4 studies from the University of Michigan [32, 51, 56, 58]; third, 3 studies from Sichuan University [47, 48, 57].

Most studies include either only female subjects [31, $32,39,41-43,46,49,51,52,54,59]$ or many more females than males $[22,23,35,45,50,53,56,58,60]$. While some studies specifically focused on patients in a narrow age-range (for example, from 20 s to 30 s) [32, 47, $48,51,52,56-59]$, others included a broader range of ages, ranging from 20 s to 50 s $[22,23,31,35,39,41-43$, $45,46,49,50,53-55,60]$. Five studies [31, 32, 51, 56, 58 included patients with myofascial TMD and 2 studies $[49,53]$ investigated patients with TMJ synovitis pain, whereas the other studies included patients with either muscle pain or synovitis pain or both. The average duration of symptoms across the studies ranged from 14 months to 12 years. Pain intensity and other pain-related characteristics were recorded using the visual analog scale [32, 35, 49-51, 53, 55, 56], numeric pain scale [39, 41-43, 46], numerical rating scale [31, 54, 59], McGill pain questionnaire $[22,23,32,35,45,50-52,56,58]$ or Graded Chronic Pain Scale [47, 48, 55, 57, 60].

Four studies $[47-49,57]$ reported that patients took no medication for TMD treatment before. Five studies $[52,53,55,59,60]$ did not report whether patients were medicated for TMD or not. Other studies reported details on individual medication status and/or asked patients to be free of pain medications before MRI scanning [22, 23, 31, 32, 35, 39, 41-43, 45, 46, 50, 51, 54, 56, 58].

\section{Quality assessment}

Quality scores for each study are reported in Additional file 1: Table S1. A total score of 9 was possible. For the study not including a control group [60], a maximum score of 7 was allowed. The majority of included studies showed a moderate to high score of methodological quality assessment. A prevalent strength of included studies was the detailed descriptions of the patient and control groups, in which the age and sex were matched, although a few studies $[23,35,45,50,52,60]$ did not report whether recruited TMD patients are free of 


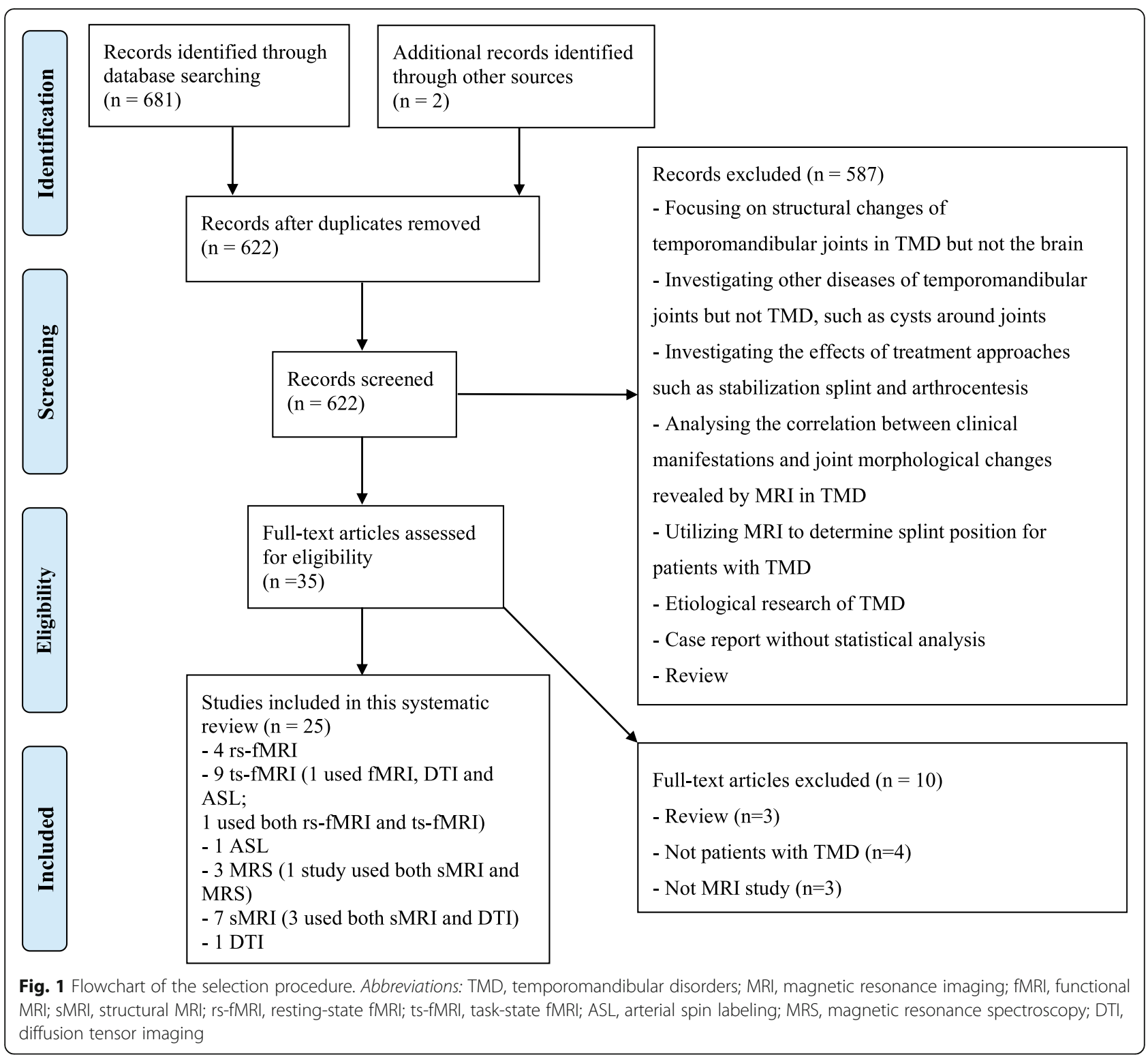

fibromyalgia or other chronic pain disorders. All studies, except 1 with 2.0 Tesla [53], used 3.0 Tesla MRI scanner.

\section{Alterations in the trigemino-thalamo-cortical pathway Alterations in the trigeminal nerve roots}

In MRI studies, patients with TMD displayed significantly lower FA [22, 42], higher MD [22] and decreased GMV [22] in the trigeminal nerve root compared with healthy controls. A negative correlation was found between the FA in the right trigeminal nerve root and TMD duration [42]. However, an earlier TMD study [45] found no diffusivity changes in the trigeminal nerve root using a manual ROI analysis.

\section{Alterations in the brainstem}

Reports of volumetric changes in the trigeminal principal sensory nucleus $(\mathrm{Vp})$ in TMD are inconsistent. Wilcox et al. [22] found decreased GMV in the ipsilateral subnucleus interpolaris and caudalis of the spinal trigeminal nucleus as well as the ipsilateral Vp in TMD patients, which was the opposite of an increased GMV in Vp reported by Younger et al. [31]. Wilcox et al. [22] suggested that the discrepancy may arise from the differences in pain duration between the patients (4.4 vs 9.7 years), possibly reflecting short-term compensatory volume increase followed by volume decrease over a longer-term course of illness [22]. In addition, another MRI study [23] using ASL detected a significant increase of blood flow in several brainstem regions in TMD 


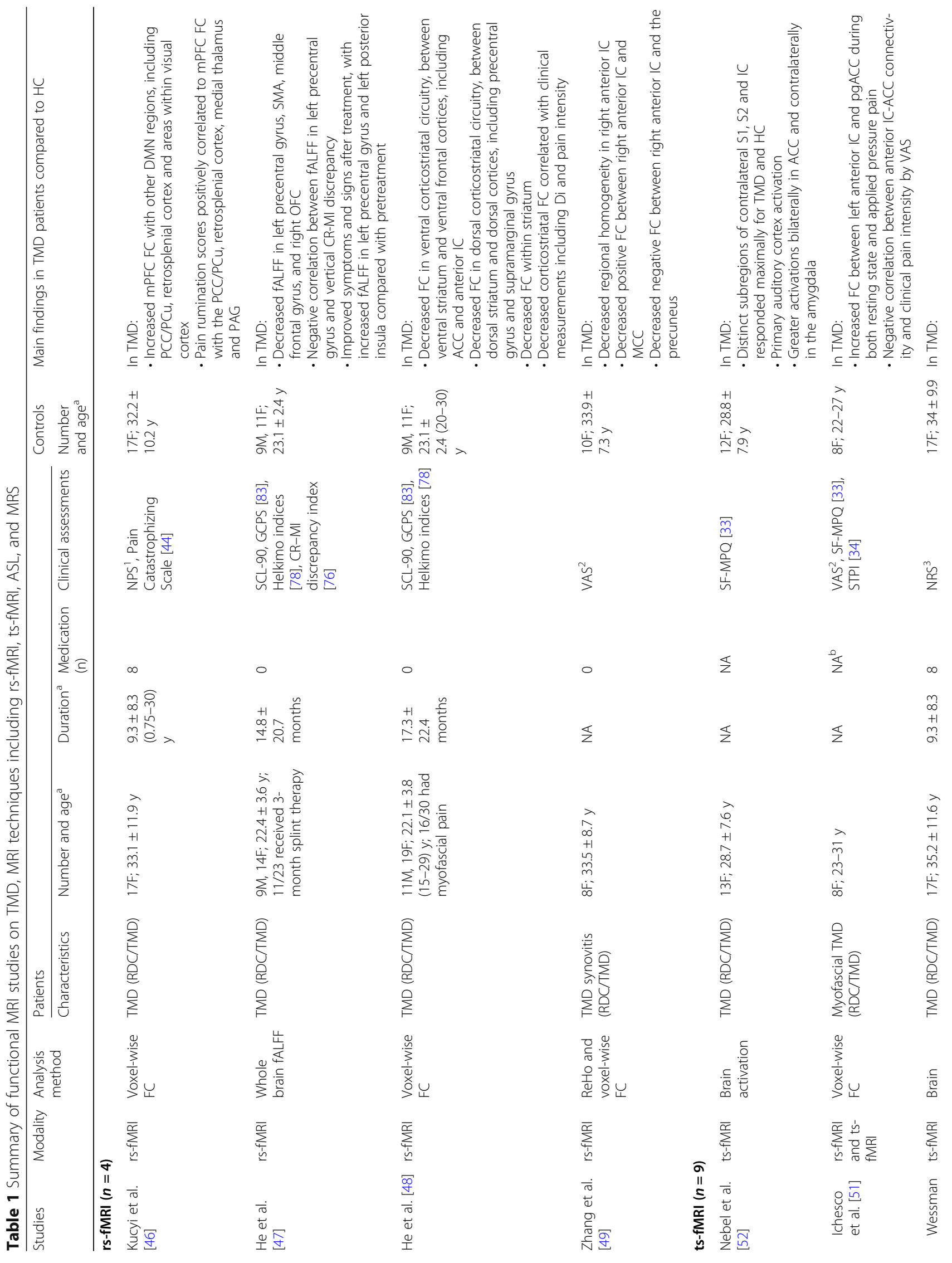




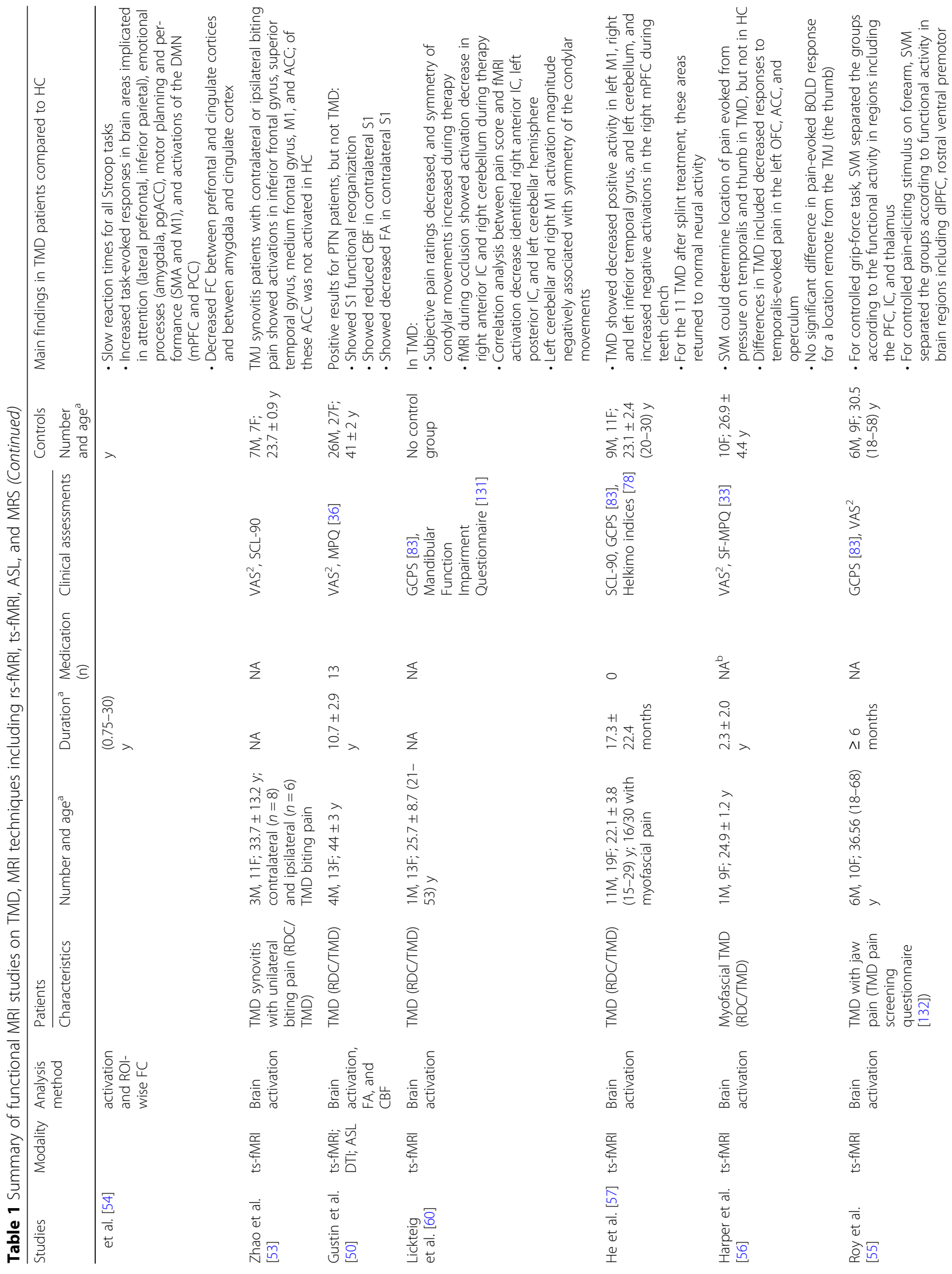




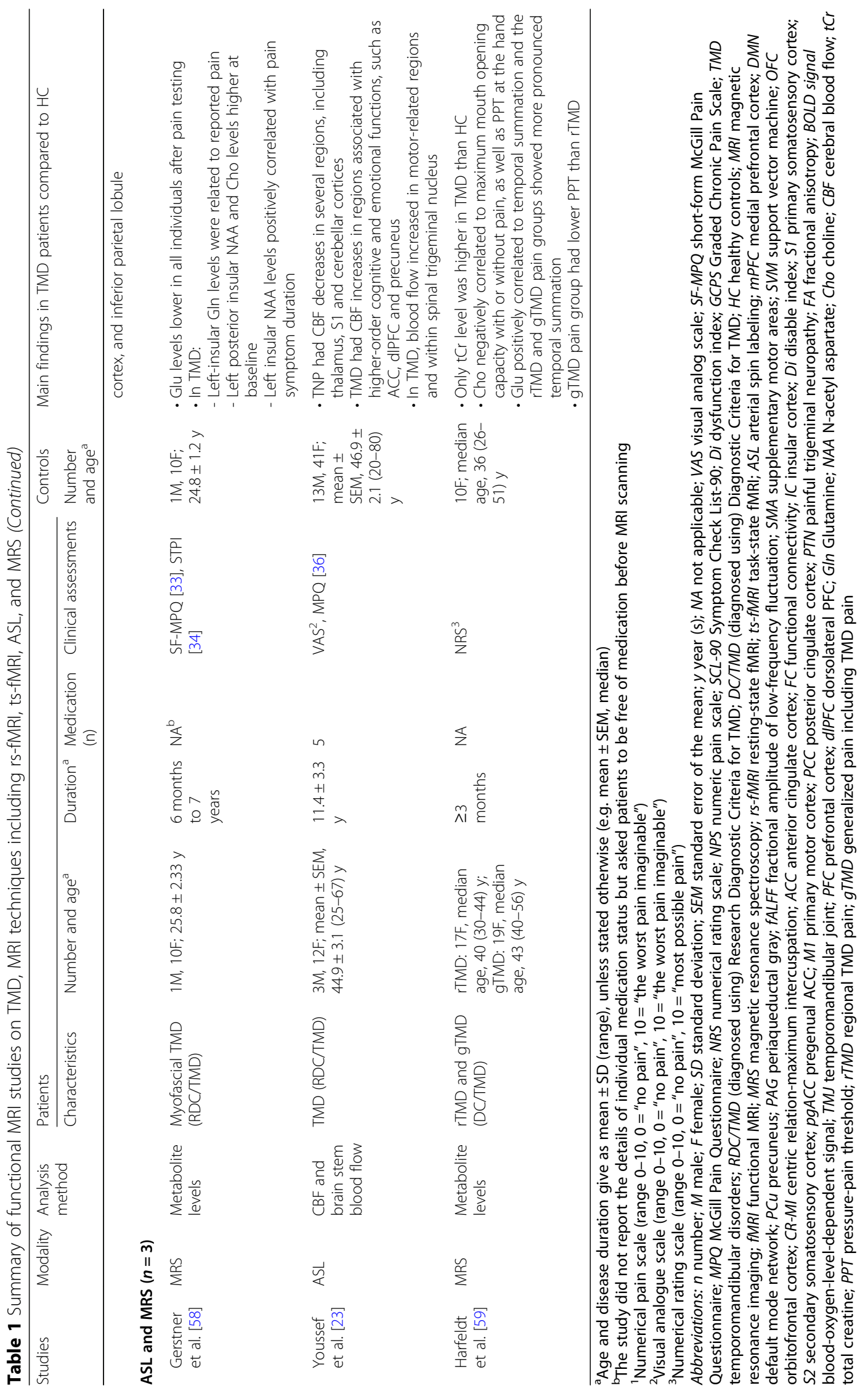




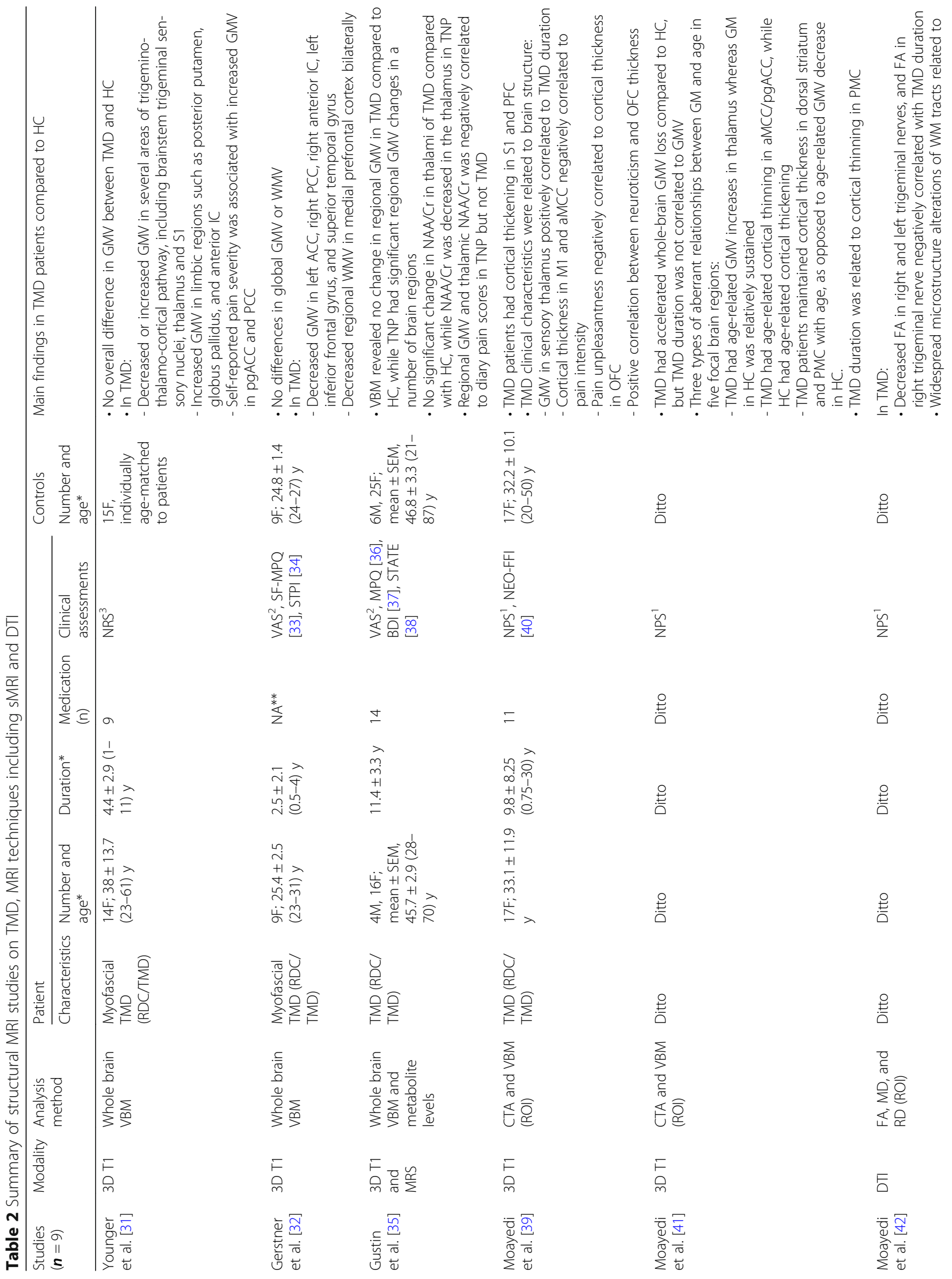


Yin et al. The Journal of Headache and Pain

(2020) 21:78

Page 9 of 20

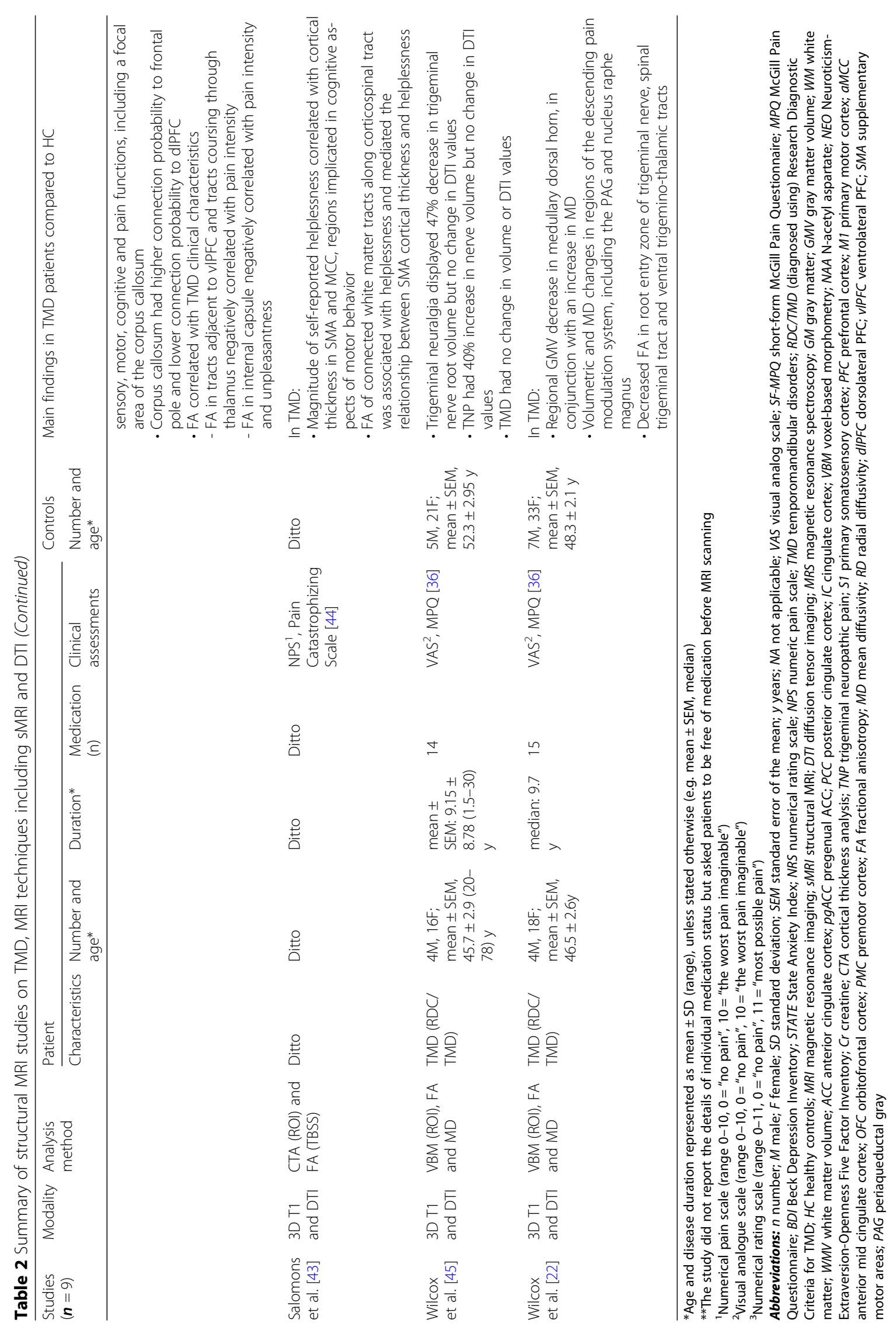


patients, including the right $\mathrm{SpVc}$, right $\mathrm{Vp}$, and rostral pons encompassing the ventral trigemino-thalamic tract.

Wilcox et al. [22] also used DTI to investigate microstructural alterations in the brainstem, focusing on the $\mathrm{SpVc}$ and pain-processing pathways. Compared with 40 pain-free controls, 22 patients with TMD showed a significant increase of MD in the ipsilateral spinal trigeminal nucleus, bilateral trigeminal nerve tract within the pons and PAG [22]. Another DTI study [42] confirmed aberrant peripheral input from the trigeminal nerve, finding lower FA in the brainstem white matter along the ascending nociceptive pathways coursing through the thalamus, internal capsule and tracts projecting to sensorimotor cortex.

\section{Alterations in the thalamus and S1}

The thalamus and $\mathrm{S} 1$ are chief projections from the trigeminal nerve system [61] and play essential roles in the thalamocortical pathway related to pain [62]. The ventral posterior thalamus receives nociceptive and other sensory information from the periphery and projects to S1 [63]. Two studies [31, 39] reported significantly increased GMV of thalamus in TMD compared to controls, and 1 found a positive correlation between the thalamus GMV and TMD duration [39]. Additionally, age-related GMV increase in the thalamus was found in TMD patients [41], compared to a weak age-related GMV decrease in healthy controls.

Anatomical MRI studies of S1 changes in TMD have generated less consistent results, with different studies reporting decreased GMV [31], increased cortical thickness [39] or no change in GMV [35]. This divergence may result from differences in pain duration, as well as the impact of medication. To investigate changes of S1 in TMD further, Gustin et al. [50] used multiple modalities of MRI (ts-fMRI, DTI, and ASL) to determine whether S1 reorganization occurred in both neuropathic (e.g. painful trigeminal neuropathy $[\mathrm{PTN}]$ ) and nonneuropathic (e.g. TMD) pain. They found that innocuous brushing of the lip, thumb and little finger resulted in similar functional activation in S1 of TMD patients and controls, and found no significant differences in FA or CBF within contralateral S1 between TMD patients and controls, while PTN patients displayed functional reorganization evidenced by reduced FA and $\mathrm{CBF}$ in $\mathrm{S} 1$ [50]. Another ASL study [23] found no significant CBF changes in the thalamus and S1 in patients with TMD, as opposed to CBF decrease in the 2 areas in PTN patients. Gustin et al. [50] suggested that the critical factor for S1 reorganization may be the constant S1 input, regardless of pain type (i.e. neuropathic vs nonneuropathic pain); the lack of increased S1 activity in TMD patients as revealed by ASL might reflect a lack of constant nociceptive input to $\mathrm{S} 1$ as a general feature of TMD patients [50].

\section{Alterations in cortical regions Altered lateral and medial pain systems}

The lateral and medial pain systems are involved in the nociceptive processing of pain and responsible for individual differences in its experience. The lateral pain system mainly carries information to the lateral thalamic nuclei which projects to S1, secondary somatosensory cortex, posterior insula and mid-cingulate cortex (MCC), etc., and is believed to encode pain intensity, laterality and somatotopy [64]. The medial pain system is implicated in mediating the more affective-motivational aspects in the experience of pain. This circuitry mainly relays information through the medial thalamic nuclei to the anterior insula and anterior cingulate cortex (ACC), integrating interoceptive input with its emotional salience [65].

The insula and cingulate cortex are the most consistently activated forebrain regions when someone experiences pain. Younger et al. [31], studying 15 female patients with myofascial TMD, first reported increased GMV in the right anterior insula and a negative correlation between self-reported pain intensity and GMV in the pregenual ACC (pgACC) and posterior cingulate cortex (PCC). Ichesco et al. [51] investigated FC between insula and cingulate cortex in 8 female patients with TMD and found elevated FC between left anterior insula and pgACC in resting state. They also showed that during a task state with an applied pressure pain as a controlled stimulus, this FC was negatively correlated with subjective pain intensity [51], i.e. patients with increased connectivity reported lower pain, suggesting compensatory brain changes to regulate pain in patients. A recent rs-fMRI study by Zhang et al. [49] found decreased regional homogeneity in the right anterior insula in $8 \mathrm{fe}-$ male patients with TMJ synovitis pain relative to 10 healthy controls. Gerstner et al. [32] reported decreased GMV in the right anterior insula, ACC, and PCC in $9 \mathrm{fe}-$ male TMD patients, suggesting that via excessive stimulation or increased compensatory inhibitory input, anatomic changes may also be observed.

In regard to MCC involved in the lateral pain system, Zhang et al. [49] reported decreased FC between MCC and anterior insula, which was negatively correlated with pain intensity in TMD patients, i.e. patients with decreased connectivity reported higher pain. MRI studies investigating TMD patients have also reported decreased FC between MCC and dorsolateral prefrontal cortex (dlPFC) [54], lower FA in MCC [42] and a negative correlation between the cortical thickness of MCC and pain intensity [39]. 
The involvement of posterior insula in TMD-related chronic pain has also been noted. Two ${ }^{1} \mathrm{H}$ MRS studies $[58,59]$ investigated neurochemistry in the posterior insula of TMD patients. MRS-detectable neurometabolites include glutamate (Glu), a major excitatory neurotransmitter contributing to the negative affect associated with pain [66], and its metabolite glutamine (Gln), both involved in complex metabolic cycles between neurons and astrocytes [67]. MRS studies have also examined Nacetyl aspartate (NAA), choline (Cho) and total creatine $(\mathrm{tCr})$, markers of other aspects of neurochemistry. Gerstner et al. [58] found a negative correlation between Gln levels in the left insula and reported pain in 11 TMD patients; NAA and Cho levels in the left posterior insula were increased compared to 11 healthy controls and NAA levels were positively correlated with the duration of pain. In addition, Harfeldt et al. [59] reported elevated $\mathrm{tCr}$ levels within the posterior insula in 17 TMD patients relative to 10 healthy controls; in the patient group, increased Cho levels correlated with a reduced capacity for mouth opening and lower pressure pain threshold on the hand, while Glu levels were positively correlated with temporal summation of the nociceptive mechanical stimulus.

\section{Altered DMN and pain modulation}

The DMN is a group of functionally interconnected brain regions known to be active when people are mind wandering and not involved in any specific task, which becomes correspondingly deactivated during goaloriented tasks [68]. Activity in this network reflects selfmonitoring activity and the processing of internal state information. In patients with TMD, Weissman-Fogel et al. [54] reported task-evoked activation in the medial prefrontal cortex (mPFC) and PCC, as well as functional dysconnections within the DMN in TMD patients performing a task with emotional interference. In addition, Kucyi et al. [46] found enhanced FC between $\mathrm{mPFC}$ and other DMN regions including $\mathrm{PCC} /$ precuneus $(\mathrm{PCu})$, retrosplenial cortex, and areas within visual cortex. Furthermore, the pain rumination scores were positively correlated with the FC between $\mathrm{mPFC}$ and $\mathrm{PCC} / \mathrm{PCu}$, retrosplenial cortex, mediodorsal thalamus, and PAG [46].

The PAG-raphe magnus system is the best-studied pathway for descending pain modulation [69]. Wilcox et al. [22] demonstrated that in TMD subjects, the PAG displayed a significant increase of $M D$ value and no GMV change, while the nucleus raphe magnus showed GMV decrease and no change in diffusivity.

A number of higher-level brain areas have also proved to be engaged in pain control, including the cingulofrontal regions, amygdala, and hypothalamus [70]. Increased CBF has been found in TMD patients in brain regions implicated in cognitive and emotional functions, including the ACC, dlPFC and PCu [23]. Patients with TMD often show impaired cognitive ability on neuropsychological testing [71, 72]. Weissman-Fogel et al. [54] demonstrated that TMD patients had aberrant brain responses while performing the attention-demanding Stroop task with cognitive and emotional interference, including reduced $\mathrm{FC}$ within two pairs of brain regions, the anterior MCC [aMCC]-dIPFC and pgACC-amygdala. Another study [39] employing sMRI reported that TMD patients had thicker cortex in the frontal pole and ventrolateral prefrontal cortex compared with healthy controls, and that cortical thickness of orbitofrontal cortex (OFC) was negatively correlated to pain unpleasantness. Additionally, cortical thickness in the left ventromedial prefrontal cortex (vmPFC, part of OFC) was positively correlated with neuroticism scores [40] in TMD patients, different from a negative correlation in healthy subjects [73].

\section{Alterations in the motor system}

Though this is less well-explored than the sensorydiscriminative and affective-motivational components of pain, there is increasing evidence supporting involvement of the motor system in patients with TMD-related pain. Wessman-Fogel et al. [54] found that TMD patients showed elevated activity in the primary motor cortex (M1) and supplementary motor areas (SMA) during the cognitive interference Stroop task. He et al. [47] identified decreased neural spontaneous function in M1 and SMA in treatment-naive TMD patients, measured by the fractional amplitude of low-frequency fluctuation (fALFF) calculated from rs-fMRI data. Centric relationmaximum intercuspation (CR-MI) discrepancy of bilateral TMJs is an indicator of the presence and severity of TMD [74, 75], and He et al. [47] found a negative correlation between the fALFF in M1 and vertical CR-MI [76] in TMD patients. Furthermore, in an sMRI study by Salomons et al., [43] self-reported helplessness in TMD patients assessed by Pain Catastrophizing Scale [44] was positively correlated with the cortical thickness of SMA, a critical region implicated in cognitive aspects of motor behavior [77], but further analysis [43] identified neither significant group difference in the cortical thickness of SMA nor correlation with pain characteristics.

In addition to M1 and SMA, the striatum has also been implicated in the motor response to pain in TMD. TMD patients with myofascial pain had increased GMV in the right putamen and right globus pallidus relative to controls [31]. TMD patients also showed sustained increased GMV in the dorsal striatum independent of TMD duration, while healthy controls had normal agerelated gray matter atrophy in this region [41], suggesting an aberrant pattern of striatal aging in TMD. In 
addition, a rs-fMRI [48] study explored the FC of the corticostriatal circuit in patients with TMD. Compared with controls, the patient group had reduced FC in the ventral corticostriatal circuitry between the ventral striatum and ventral-frontal cortices (ACC and anterior insula), in the dorsal corticostriatal circuitry between dorsal striatum and dorsal cortices (precentral gyrus and supramarginal gyrus), and also within the striatum [48]. Exploratory analysis found associations between decreased corticostriatal FC and clinical variables of overall clinical dysfunction measured by Helkimo indices [78] and pain intensity [48].

\section{Altered brain activations in response to mechanical stimuli}

Previous studies found that patients with TMD had abnormal vibrotactile sensibility on the face, with elevated detection threshold [79] and impaired frequency discrimination [80], indicating a disruption of the somatosensory system in TMD. Compared with healthy controls, with the stimulation of low frequency and innocuous vibration of the index finger, patients with TMD displayed greater activations in bilateral ACC and contralateral amygdala [52], 2 critical regions implicated in the emotional aspect of pain [81, 82]. In addition, 16 TMD synovitis patients with biting pain showed elevated activation of ACC during clenching tasks compared with 14 controls, and the activation of ACC was found to be associated with higher levels of psychological distress in patients [53].

Multivariate analysis techniques have begun to be used to identify the central mechanisms underlying pain processing in TMD. Roy et al. [55] investigated neural activity during a grip-force task and a pain-eliciting forearm thermal stimulus in TMD patients with chronic jaw pain [83] and controls, using multivariate analyses to identify brain regions whose functional activity could discriminate between the groups. By using multivariate analyses, in the motor control task, increased activity in brain areas including PFC, insula, and thalamus could distinguish the patients from controls with a mean test for the area under the receiver operator characteristic curve (AUC) of 0.8, although the parameters of grip-force production were similar in both groups [55]. In the pain task, there was no significant difference in stimulus intensity and pain perception between groups, but the functional activations of dlPFC, ventral premotor cortex, and inferior parietal lobule could distinguish the groups with a mean test AUC of 0.85 [55].

Harper et al. [56] explored the differences in brain activity evoked by subjectively equated pain originated from temporalis (a clinically painful region) and thumb (a remote asymptomatic area) in patients with TMD and controls. The support vector machine (SVM) method was able to distinguish between the neural activations in areas with cognitive valuation of pain, including the left OFC, ACC, and operculum in response to temporalisevoked pain and thumb-evoked pain in TMD patients with an accuracy of $75 \%$, but not in healthy controls (55\%) [56].

\section{Brain changes after splint therapy}

Lickteig et al. [84] first used fMRI to record cerebral activation changes of a single TMD patient before and after splint therapy (individually optimized, applied for 11 nights and partial days) and found decreased brain activity (in bilateral sensorimotor regions and other areas such as left posterior insula) during occlusion (both on natural teeth and splint) after treatment compared to the untreated baseline. The same group performed a longitudinal fMRI study to assess the effect of 2-week splint therapy on 14 patients with TMD [60]. After splint treatment the patients showed reduced activations in the right anterior insula and right cerebellar hemisphere during occlusion, accompanied by relief of subjective pain and increased symmetry of condylar movements. Correlation analysis identified an association between reduced pain scores and attenuated activations in the right anterior insula, left posterior insula and left cerebellar hemisphere, as well as between improved condylar movements and decreased activations in the left cerebellar and right M1 [60].

He et al. [47] used rs-fMRI to investigate the spontaneous brain activity of 23 TMD patients with CR-MI discrepancy and assessed the therapeutic effect of maxillary stabilization splint. Eleven out of 23 patients received 3 months splint therapy, showing improved signs and symptoms and more stable condylar position; the fALFF in left M1 and left posterior insula, which had been decreased before treatment compared to healthy controls, recovered to the normal level [47]. Another study [57] explored neural activation in TMD patients during a clenching task before and after stabilization splint therapy. Compared with the control group, TMD patients before treatment exhibited decreased positive activations in cerebral areas associated with motor and cognitive functions (including the left M1, bilateral inferior temporal gyrus and left cerebellum) and elevated negative activations in the DMN (right mPFC). Neural activity in these cortical regions normalized after splint therapy, accompanied by improvement of TMJ status [47].

\section{Discussion}

In this review, 25 original MRI studies were retrieved to investigate the neuro-pathophysiological manifestations of TMD-related pain. Notably, our findings provide evidence for both peripheral and central neural basis for pain in TMD (briefly summarized in Fig. 2). The lack of 


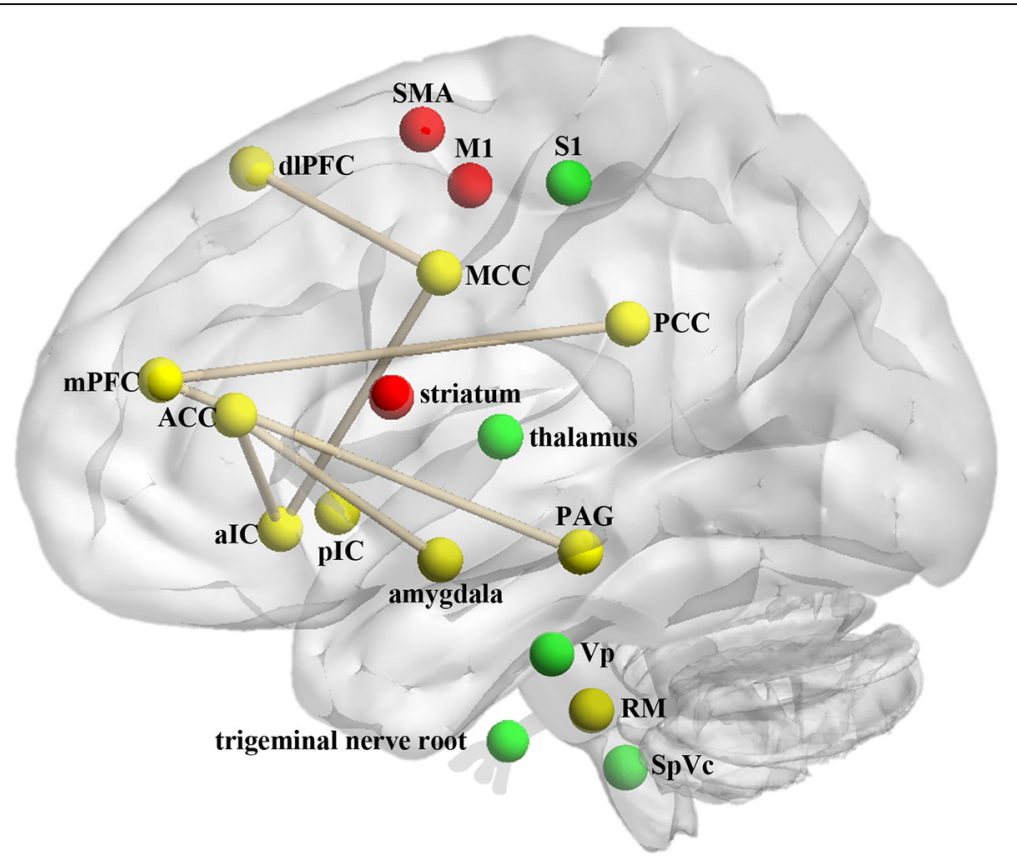

Fig. 2 Schematic representation of main brain regions with altered structure and function involved in TMD related-pain. Green balls represent the areas in the classic trigemino-thalamo-cortical system. Red balls are in the motor system. Yellow balls are the brain cortical regions implicated in pain perception and pain modulation. Brain regions with altered functional connectivity in TMD are connected with lines in khaki. Abbreviations: SMA, supplementary motor areas; dIPFC, dorsolateral prefrontal cortex; M1, primary motor cortex; S1, primary somatosensory cortex; MCC, midcingulate cortex; mPFC, medial prefrontal cortex; ACC, anterior cingulate cortex; PCC, posterior cingulate cortex; alC, anterior insular cortex; pIC, posterior insular cortex; PAG, periaqueductal gray; Vp, trigeminal principal sensory nucleus; RM, raphe magnus; SpVc, spinal tract subnucleus caudalis

large sample size studies and the variety of methodological approaches complicate the process of synthesizing findings to reach broad conclusions and may explain some discrepancies in results between studies. The following discussion will provide a detailed interpretation of the findings and their clinical implications in a broader context.

\section{Implications of findings in the trigemino-thalamo-cortical pathway}

The classic trigemino-thalamo-cortical pathway is responsible for the sensation of oral and maxillofacial regions [85]. Current evidence reveals brain structural and functional alteration in key nodes within this ascending trigemino-thalamo-cortical pathway in TMD.

Diffusivity changes observed in the trigeminal nerve root $[22,42]$ may reflect long-term microstructural alterations of the nerve, a manifestation of microstructural changes in response to increased peripheral nociceptive input. The reported discrepancy of diffusivity changes in the trigeminal nerve $[22,45]$ results from methodological differences between the two studies (with the same samples). The earlier study [45] used manual ROI analysis and the results could be affected by partial volume effects given the small size of the trigeminal nerve root, while the deterministic tractography used in the later study [22] provided greater sensitivity and a more accurate estimate of diffusion properties of white matter tracts [86]. Additionally, the GMV decrease and MD increase in SpVc [22] may reflect glia shrinkage/atrophy or neuronal loss [87] and a reduction in dendritic density [88], while the elevated blood flow in SpVc [35] could be a compensatory response of increased neural activity to structural reductions. As such, the neural structural and blood flow changes in the initial part of the trigeminal system (the root entry zone and $\mathrm{SpVc}$ ) indicate that the established hyperexcitability of the nociceptive processing pathways is critical for the altered perception and maintenance of pain in TMD [89]. On the other hand, increased GMV and aberrant age-related GMV changes in the thalamus may also be due to persistent trigeminal nociceptive input, which further contributed to the hyperalgesia of TMD by enhanced facilitating trigeminal sensory information from the thalamus to S1.

However, the structural changes of S1 are somewhat less consistent. Gustin et al. [50] found no functional reorganization in the S1 of TMD patients and no significant difference in FA or CBF within S1 compared with healthy controls. This may not necessarily rule out alterations within the trigemino-thalamo-cortical pathway in 
the TMD patients examined by Gustin et al. [50], as the authors did not investigate the structural and functional changes of other critical areas within the pathway, especially the $\mathrm{SpVc}$ and thalamus. Therefore, further MRI studies focusing simultaneously on the $\mathrm{SpVc}$, thalamus, and $\mathrm{S} 1$ and examining both brain anatomy and function are required to resolve the issue of inconsistencies of S1 changes in TMD patients. If structural and functional alterations were found only in SpVc and/or thalamus, but not S1, it would be interesting to investigate how TMD patients deal with increased nociceptive inputs while showing intact S1. Alternatively, more refined measurements and larger sample studies may provide a clearer picture of alterations that may occur in S1 in TMD patients.

\section{Implications of cortical findings Altered lateral and medial pain systems}

The ACC and anterior insula are two important regions responsible for encoding the emotional and motivational aspects of pain. Despite some inconsistencies, current structural and functional findings in these two structures in TMD patients [31, 32, 49, 51, 53] support the role of abnormal medial pain system in nociceptive processing, demonstrating the emotional sensory signals related to pain in TMD patients.

Zhang et al. [49] found decreased FC between MCC and anterior insula in TMD patients, while Ichesco et al. [51] reported increased FC between pgACC and anterior insula. FC between anterior insula and different regions of cingulate cortex may represent different brain functional alterations in different disease severity of TMD, since the former group [49] studied patients with severe open-mouth pain ( $\geq 5$ on the visual analog scale), while the latter [51] recruited patients who were only mildly affected by TMD. We noted that the reduced FC between MCC and anterior insula and the increased FC between pgACC and anterior insula were both negatively correlated with pain intensity of TMD patients, so that these two alterations in FC may represent maladaptive alterations resulted from pain in TMD or compensatory brain changes for pain regulation, respectively.

The posterior insula of TMD patients showed cellular and molecular changes in 2 neurochemical studies [58, 59]. The positive correlation between the NAA levels in left posterior insula and duration of TMD pain suggested a time-dependent neuronal or axonal proliferation in response to pain [58], as NAA is considered as a biomarker of neuronal health and synaptic integrity [90]. In addition, since $\mathrm{tCr}$ can be considered as a biomarker of cell energetics [90], the increased $\mathrm{tCr}$ levels in the posterior insula in TMD patients [59] may indicate a state of neuroinflammation or cellular hyperactivity which has been proposed to be implicated in chronic pain [91].

\section{Dysfunctional DMN and pain modulation}

Previous studies have suggested that dysfunction of the DMN may be related to cognitive and behavioral deficits observed in patients with chronic pain [92]. In TMD patients, task-related activation, rather than typical taskrelated deactivation in DMN areas including the $\mathrm{mPFC}$ and PCC, has been observed [54]. PCC can be activated by emotionally salient stimuli and is involved in contextualizing painful stimuli [93]. The increased activation of PCC may indicate increased spontaneous pain in patients when completing cognitive tasks with emotional interference since it had greater emotional effects on patients than controls. Accordingly, mPFC was recruited during the emotion provoking task to mediate antinociceptive effects relevant to its role in descending pain modulation [94]. Moreover, given that $\mathrm{MPFC}$ and PCC are 2 functionally connected regions implicated in attention toward introspective thoughts $[95,96]$ which is required for coping with pain, their abnormal activation during task may reflect a nocifensive mechanism for patients to relieve pain by focusing on internal thoughts, which in turn leads to slower behavioral responses.

Pain rumination is perseverative negative thinking about pain. Kucyi et al. [46] linked dysfunctional DMN with pain rumination in TMD and suggested that individuals with high pain rumination measured by Pain Catastrophizing Scale [44] had particularly enhanced FC between components of the DMN including MPFC and $\mathrm{PCC} / \mathrm{PCu}$. The positive correlation between pain rumination and FC of mPFC with mediodorsal thalamus may reflect patients' persistent attempts to regulate pain, since mediodorsal thalamus is associated with the affective and emotional aspects of pain [97]. In addition, pain rumination was positively correlated with $\mathrm{FC}$ between $\mathrm{MPFC}$ and PAG, which may account for differential abilities in pain modulation given the prominent role of PAG in descending pain modulation. Since healthy controls had no such a correlation, Kucyi et al. [46] suggested that the degree to which chronic pain alters the normal function of these circuits may depend on how much patients ruminate, supporting the critical role of pain-related cognition in TMD-related brain alterations.

As a key region of the endogenous pain inhibitory system, PAG is well-positioned to modulate pain perception for interactions between ascending inputs from peripheral tissue and descending projections from brain regions (e.g., $\mathrm{ACC}$ and $\mathrm{mPFC}$ ), and shows anatomical alterations in other chronic pain conditions $[98,99]$. Since nucleus raphe magnus serves as the recipient in the descending analgesic pathway of PAG [100], reduced dendritic spine numbers in the PAG may alter the descending input to raphe magnus, variably impacting the efficiency of endogenous analgesia. Thus, the dysfunction of the PAG-raphe magnus descending 
modulatory pathway may shed light on the mechanism underpinning the central sensitization to pain in TMD patients.

It has been established that pain can induce attentional biases [101], and attentional manipulation is able to modulate the perception of pain [102-104]. This highlights the importance of studying brain attentional systems in relation to pain in TMD. In the study by Weissman-Fogel et al. [54], TMD patients showed slower task responses, with reduced FC within two pairs of brain regions (aMCC-dlPFC and pgACC-amygdala). The amygdala is critically implicated in the experience and expression of emotion [81, 82], and pgACC can modulate the reactivity of amygdala when confronted with emotionally salient stimuli $[105,106]$. aMCC and dIPFC are highly functionally connected; the activation of aMCC can capture attention to adjust behavior [107, 108], which is mediated by a top-down process that engages dlPFC. Therefore, reduced connections between these two pairs of structures in TMD patients may suggest an influence of their chronic pain onto attentional and emotion networks, which results in attenuated and unsynchronized recruitment of attention-processing areas and consequently slower behavioral responses.

Neuroticism is one of the 'Big Five' personality traits and individuals with high neuroticism are more likely to interpret common situations as threatening and minor frustrations as hopeless. It is a personality feature associated with increased levels of current and future anxiety and distress $[109,110]$. High neuroticism is also correlated with heightened pain-related suffering [111] and sensitivity [112]. In TMD patients, Moayedi et al. [39] found a positive correlation between cortical thickness in vmPFC and neuroticism relative to a normal negative correlation in healthy controls [73]. While neuroticism is generally a stable trait, it also can vary with level of anxiety and distress associated with physical and psychiatric illness. Thus, observations of associations with neuroticism suggest that greater levels of distress and anxiety in TMD are associated with gray matter changes in the vmPFC of TMD patients, which may result from or contribute to reductions in the brain's capacity for pain control.

\section{Motor aspects of TMD-related pain}

Neuroimaging studies in patients with TMD pain have reported structural and functional changes in the motor system, including M1, SMA, and striatum. Persistent pain can inhibit protective movement and impair motor performance due to maladaptive neuroplasticity in the motor cortex [113, 114]. Increased activity in M1 and SMA during cognitive interference Stroop task in TMD patients [54] may indicate a possible compensatory mechanism to recruit motor areas to meet elevated demands for motor planning and performance.

Uncontrollable stressors like persistent chronic pain can lead to learned helplessness, a maladaptive response featured by reduced motor escape behaviors and deficits in motivation and learning [115]. The SMA has been implicated in motor planning and pain processing when pain is perceived to be uncontrollable $[77,116]$. In the study by Salomons et al. [43], the cortical thickness in SMA did not differ between groups or show any significant correlation with chronic pain characteristics, indicating that the positive correlation between cortical thickness and helplessness was not derived from the cumulative effects of pain. Rather, helplessness in patients with painful TMD may be a function of interactions between predisposing factors, i.e. structural characteristics of nervous systems implicated in motor planning and function and persistent exposure to uncontrollable chronic pain.

As the major component of basal ganglia, striatum receives input from cortical regions and thalamic nuclei, and sends output to other structures of basal ganglia, serving as a critical site where cognitive, motor and limbic signals from other brain regions overlap and are integrated $[117,118]$. Findings of increased GMV in the right putamen and right globus pallidus [31], which contain neurons responsive to nociceptive stimuli and function for preparing behavioral responses [119], suggest a possible somatotopic reorganization or perhaps synaptic hypertrophy associated with sustained TMD pain. Correlations between reduced corticostriatal FC and clinical measurements [48] highlight the critical role of the striatum and corticostriatal loops in the motor response to pain.

\section{Altered brain activations in response to mechanical stimuli}

Compared with healthy controls, TMD patients display perceptual amplification of pressure stimulation across a wide range of physical intensities, from gentle and innocuous to strongly painful [120]. fMRI allows the detection of differences in brain function in processing external stimuli between TMD patients and healthy subjects. The differences in the location and magnitude of brain activation responsive to innocuous vibrotactile stimulation between patients and healthy controls and the activation of pain-related regions by innocuous input, as reported by Nebel et al. [52], may reflect the dysfunction of the somatosensory system in individuals with TMD. Findings of Harper et al. [56] using SVM provided further support for the involvement of aberrant central pain processing in patients with TMD pain. The ability of SVM to discriminate the location of noxious stimuli only in TMD patients by different functional activity in 
regions including $\mathrm{OFC}, \mathrm{ACC}$, and operculum indicate somatotopic-dependent differences in pain processing, reflecting regional differences of brain activation related to the cognitive evaluation of pain between TMD patients and healthy subjects.

Although TMD, like many chronic pain conditions, is accompanied by motor impairment, few MRI studies have directly investigated neural activity during a motor task in chronic pain. Roy et al. [55] first investigated brain response during a grip force task and a thermal pain stimulus in TMD patients with chronic jaw pain and healthy controls, and identified brain regions with significantly different functional activities which could separate the groups. They found that TMD patients with chronic jaw pain had different brain processing of motor- and pain- related stimulus compared with healthy controls, which supports the idea that chronic pain is correlated with task-specific brain alterations in the transformation of sensory input to motor activity and to pain perception.

\section{Neural therapeutic mechanism of splint therapy}

Treatment for TMD has been focused on alleviating the main symptoms, especially chronic pain, and the palliative approaches which prevail over surgery have relatively satisfactory clinical outcomes $[3,121,122]$. Splint therapy is one of the commonest conservative treatments for TMD. A recent meta-analysis evaluating various oral orthotic appliances concluded that hard stabilization splints, when adjusted properly, have good evidence of modest efficacy in reducing TMD-related pain relative to non-occluding appliances and no treatment [123]. Stabilization splints are designed to improve the functional movements of TMJs by eliminating occlusal interferences and removing the impact of maximization intercuspation occlusion on joint position, and also improve masticatory function by reducing abnormal muscular activity $[124,125]$. It has also been hypothesized that splints can increase patients' cognitive awareness of oral parafunctional habits, thus altering proprioceptive input and central motor areas implicated in masticatory function $[126,127]$.

The recent fMRI studies by Lickteig et al. [60, 84] and $\mathrm{He}$ et al. [47, 57] revealed the neural mechanisms of splint therapy, finding that it may work partly by eliciting neuroplastic recovery of affected brain regions. Lickteig et al. [60, 84] found decreased activations in brain regions accompanied by improved clinical symptoms after splint treatment compared with pretreatment levels, but there was no control group to assess whether they recovered to normal. On the other hand, He and coworkers $[47,57]$ found that TMD patients exhibited recovery of previously decreased baseline neural activity after splint therapy, compared with healthy controls.
This discrepancy may result from differences in patients' age, medication usage and splint design. Future MRI studies investigating the therapeutic effects of splint therapy for TMD are expected to identify a general mode of splint impact on the brain of TMD patients.

\section{Limitations}

The present review, and the field of TMD brain imaging research, have several limitations. The first important consideration is the difference in pain origins and TMD patients recruited in studies. Since TMD is an umbrella term encompassing a number of painful conditions involving masticatory muscles, TMJ and associated structures [128], the large variability in disease characteristics of patient groups precludes drawing a definite conclusion about brain changes in TMD with a specific type and pain origin. Studies seeking to identify subgroups of patients in large samples of TMD patients are needed to resolve this important issue.

Another consideration is the gender of participants. The sex ratio of patients seeking medical advice has been reported as ranging from 3:1 (women: men) to as high as 9:1 [129, 130]. Accordingly, there were many more female subjects in the studies discussed in this review. However, no neuroimaging studies of TMD have specifically targeted gender differences or investigated the neural basis of such high morbidity in women.

A third factor that impacts neuroimaging studies of TMD is the potential impacts of analgesics, which may reduce some functional alterations and induce other functional changes that might impact the experience of persistent TMD-related pain. Studying untreated TMD patients at illness presentation, as in other disorders, would be a strategy to advance clarity of illness related alterations.

Fourth, causal relationships are inherently difficult to establish in cross-sectional studies. Among the brain alterations in TMD patients, differentiating features that are the consequence of persistent pain or represent compensation efforts to reduce pain can not be confidently determined at this point, which requires further research. Moreover, findings from existing studies suggest the possibility that certain structural characteristics of the brain associated with levels of anxiety and distress and helplessness may either make some people more vulnerable to developing TMD or represent responses to TMJ-related pain with their own impact on brain anatomy and function.

Last, given that TMD is a multifactorial disease, understanding the personal difference in central sensitization is critical for choosing proper clinical management. Therefore, the combination of neuroimaging techniques and machine learning algorithms such as SVM may serve to benefit future studies of the central 
mechanisms underlying TMD pain, and eventually facilitate clinical practice by providing new diagnostic strategies, as well as objective measures of therapeutic efficacy.

\section{Conclusion}

This article systematically reviewed the existing literature investigating brain changes in TMD patients for revealing the pathogenic mechanisms or consequences of TMD-related pain. MRI neuroimaging techniques have provided a deeper understanding of what happens to brain structure and function in patients with TMD, suggesting both peripheral and central neural basis for the most common conditions of TMD, i.e. hyperexcitability to external stimuli and disrupted pain perception and pain modulation. This review can enhance our understanding of the pathophysiology underlying symptoms of TMD-related pain as targets for treatment development and planning. Primary novel observations include:

- There are structural and functional changes in the classic trigemino-thalamo-cortical system, including peripheral trigeminal nerve roots, brainstem $(\mathrm{SpVc}$ and $\mathrm{Vp}$ in particular), thalamus, and $\mathrm{S} 1$, which provides support for a peripheral origin of TMD. Specifically, the results of increased GMV in the thalamus were consistent, while whether there are alterations in S1 in TMD patients remains to be clarified.

- There are alterations in several cortical regions implicated in pain perception and pain modulation in TMD. Neurochemical alterations are identified in the posterior insula. The altered FCs among anterior insula, pgACC and MCC are correlated with pain intensity. The dysfunctional DMN in TMD patients is characterized by reduced FC in $\mathrm{mPFC}-\mathrm{PCC} / \mathrm{PCu}$ and mPFC-PAG. TMD related pain-attention interaction is mediated by reduced FC in aMCC-dlPFC and pgACC-amygdala. Structural changes in the PAG-raphe magnus pathway may impair the efficiency of the endogenous pain inhibitory system of TMD patients.

- Regional functional brain changes in M1 and SMA, as well as increased GMV and decreased FC in the striatum, indicate the compensatory changes or maladaptive neuroplasticity of the motor system in patients with TMD pain.

- TMD patients displayed different brain activations in the fronto-insulo-thalamo-parietal network under both innocuous and painful stimulus compared with healthy controls, reflecting the involvement of aberrant central pain processing in TMD. Multivariate analysis techniques like SVM may help distinguish the subtypes of TMD patients, i.e. identify whose pain has a more peripheral or a central etiology, which could be clinically useful for optimal treatment.

- Splint therapy can impact the neural function of brain regions in TMD patients, accompanied by improved clinical symptoms, suggesting a central mechanism underlying the therapeutic effects of splint therapy

\section{Supplementary information}

Supplementary information accompanies this paper at https://doi.org/10. 1186/s10194-020-01131-4.

Additional file 1: Table S1. The checklist of quality assessment for included studies in the present systematic review.

Additional file 2: Table S2. Regions with significant group differences between patients with TMD and controls reported in functional MRI studies included in the present systematic review. Table S3. Regions with significant group differences between patients with TMD and controls reported in structural MRI studies included in the present systematic review.

\section{Abbreviations}

TMD: Temporomandibular disorders; TMJ: Temporomandibular joint; RDC/ TMD: Research Diagnostic Criteria for TMD; DC/TMD: Diagnostic Criteria for TMD; MRI: Magnetic resonance imaging; DTI: Diffusion tensor imaging; FA: Fractional anisotropy; MD: Mean diffusivity; fMRI: Functional MRI; tsfMRI: Task-state fMRI; rs-fMRI: Resting-state fMRI; ASL: Arterial spin labeling; MRS: Magnetic resonance spectroscopy; SpVc: Spinal tract subnucleus caudalis; ACC: Anterior cingulate cortex; mPFC: Medial prefrontal cortex; PAG: Periaqueductal gray; S1: Primary somatosensory cortex; MCC: Midcingulate cortex; GMV: Gray matter volume; Vp: Trigeminal principal sensory nucleus; PTN: Painful trigeminal neuropathy; CBF: Cerebral blood flow; FC: Functional connectivity; PCC: Posterior cingulate cortex; M1: Primary motor cortex; pgACC: Pregenual anterior cingulate cortex;

SMA: Supplementary motor areas; Glu: Glutamate; GIn: Glutamine; NAA: Nacetyl aspartate; Cho: Choline; tCr: Total creatine; dIPFC: Dorsolateral prefrontal cortex; DMN: Default mode network; aMCC: Anterior mid cingulate cortex; OFC: Orbitofrontal cortex; vmPFC: Ventromedial prefrontal cortex; PCu: Precuneus; CR-MI Discrepancy: Centric relation-maximum intercuspation discrepancy; SVM: Support vector machine; fALFF: Fractional amplitude of low-frequency fluctuation; AUC: Area under the receiver operator characteristic curve

\section{Acknowledgements}

Not applicable.

\section{Authors' contributions}

FL, QYG, and SC conceived the study. YYY and SSH designed the research and drafted the manuscript. $Y Y Y$ and $S S H$ conducted literature research. $Y Y Y$, SSH, JCX, WFY, QL, JYL, LKL, GJK and JAS contributed to data acquisition, analysis and interpretation. GJK, JAS, FL, QYG, and SC critically revised the manuscript. All authors have approved the final manuscript.

\section{Funding}

This study was supported by the National Natural Science Foundation (Grant Nos. 81621003, 81671021, 81621003, 81820108018 and 81400548) of China. Dr. Gong would like to acknowledge the support from the Program for Changjiang Scholars and Innovative Research Team in University (PCSIRT, Grant No. IRT16R52) of China and the Functional and Molecular Imaging Key Laboratory of Sichuan Province (FMIKLSP, Grant: 2019JDS0044). Dr. Li would like to acknowledge the support from the Sichuan Science and Technology Program (2019YJ0098), Science and Technology Project of the Health Planning Committee of Sichuan (18ZD035), Technology Foundation for the Selected Returned Overseas Chinese Scholars (Sichuan Provincial Human Resources and Social Security Department, [2018]145-19), and Fundamental Research Funds for the Central Universities (2018SCUH0011), National Key R\&D Program of China (NO.2018YFA0108604). 


\section{Availability of data and materials}

All data and articles supporting the conclusions of this systematic review are included within the systematic review.

\section{Ethics approval and consent to participate}

Not applicable.

\section{Consent for publication \\ Not applicable.}

\section{Competing interests}

The authors declare no competing financial interests.

\section{Author details}

${ }^{1}$ Huaxi MR Research Center (HMRRC), Department of Radiology, West China Hospital of Sichuan University, Chengdu 610041, Sichuan, People's Republic of China. ${ }^{2}$ State Key Laboratory of Oral Diseases, National Clinical Research Center for Oral Diseases, Department of Orthodontics, West China School of Stomatology, Sichuan University, Chengdu 610041, Sichuan, People's Republic of China. ${ }^{3}$ Psychoradiology Research Unit of Chinese Academy of Medical Sciences, Functional and Molecular Imaging Key Laboratory of Sichuan Province, West China Hospital of Sichuan University, Chengdu 610041, Sichuan, People's Republic of China. ${ }^{4}$ Liverpool Magnetic Resonance Imaging Centre (LiMRIC) and Institute of Ageing and Chronic Disease, University of Liverpool, Liverpool, UK. ${ }^{5}$ Department of Psychiatry, University of Cincinnati, Cincinnati, OH, USA.

\section{Received: 27 February 2020 Accepted: 25 May 2020}

Published online: 19 June 2020

\section{References}

1. Okeson JP, de Leeuw R (2011) Differential diagnosis of temporomandibular disorders and other orofacial pain disorders. Dent Clin N Am 55(1):105-120

2. Luther F (2007) TMD and occlusion part I. damned if we do? Occlusion: the interface of dentistry and orthodontics. Br Dent J 202(1):E2 discussion 38-39

3. Scrivani SJ, Keith DA, Kaban LB (2008) Temporomandibular disorders. N Engl J Med 359(25):2693-2705

4. John MT, Reissmann DR, Schierz O et al (2007) Oral health-related quality of life in patients with temporomandibular disorders. J Orofac Pain 21(1):46-54

5. Dahlstrom L, Carlsson GE (2010) Temporomandibular disorders and oral health-related quality of life. A systematic review. Acta Odontol Scand 68(2):80-85

6. Dworkin SF (1994) Perspectives on the interaction of biological, psychological and social factors in TMD. J Am Dent Assoc 125(7):856-863

7. Dworkin SF, Massoth DL (1994) Temporomandibular disorders and chronic pain: disease or illness? J Prosthet Dent 72(1):29-38

8. Ohrbach R, Dworkin SF (1998) Five-year outcomes in TMD: relationship of changes in pain to changes in physical and psychological variables. Pain 74(2-3):315-326

9. Hagberg C (1991) General musculoskeletal complaints in a group of patients with craniomandibular disorders (CMD). A case control study. Swed Dent J 15(4):179

10. Yap AU, Chua EK, Dworkin SF et al (2002) Multiple pains and psychosocial functioning/psychologic distress in TMD patients. Int J Prosthodont 15(5): 461-466

11. Plesh O, Wolfe F, Lane N (1996) The relationship between fibromyalgia and temporomandibular disorders: prevalence and symptom severity. J Rheumatol 23(11):1948-1952

12. Aaron LA, Burke MM, Buchwald D (2000) Overlapping conditions among patients with chronic fatigue syndrome, fibromyalgia, and temporomandibular disorder. Arch Intern Med 160(2):221-227

13. Sarlani E, Greenspan JD (2005) Why look in the brain for answers to temporomandibular disorder pain? Cells Tissues Organs 180(1):69-75

14. Fernandez-de-las-Penas C, Galan-del-Rio F, Fernandez-Carnero J et al (2009) Bilateral widespread mechanical pain sensitivity in women with myofascial temporomandibular disorder: evidence of impairment in central nociceptive processing. J Pain 10(11):1170-1178

15. Sarlani E, Greenspan JD (2003) Evidence for generalized hyperalgesia in temporomandibular disorders patients. Pain 102(3):221-226

16. Kupers $\mathrm{R}$, Kehlet $\mathrm{H}$ (2006) Brain imaging of clinical pain states: a critica review and strategies for future studies. Lancet Neurol 5(12):1033-1044
17. Albanese MC, Duerden EG, Rainville P et al (2007) Memory traces of pain in human cortex. J Neurosci 27(17):4612-4620

18. Woolf CJ, Salter MW (2000) Neuronal plasticity: increasing the gain in pain. Science 288(5472):1765-1769

19. Davis KD, Moayedi M (2013) Central mechanisms of pain revealed through functional and structural MRI. J Neurolmmune Pharmacol 8(3):518-534

20. Cauda F, Palermo S, Costa T et al (2014) Gray matter alterations in chronic pain: a network-oriented meta-analytic approach. Neuroimage Clin 4:676-686

21. Smallwood RF, Laird AR, Ramage AE et al (2013) Structural brain anomalies and chronic pain: a quantitative meta-analysis of gray matter volume. J Pain 14(7):663-675

22. Wilcox SL, Gustin SM, Macey PM et al (2015) Anatomical changes within the medullary dorsal horn in chronic temporomandibular disorder pain. Neuroimage 117:258-266

23. Youssef AM, Gustin SM, Nash PG et al (2014) Differential brain activity in subjects with painful trigeminal neuropathy and painful temporomandibular disorder. Pain 155(3):467-475

24. Lin CS (2014) Brain signature of chronic orofacial pain: a systematic review and meta-analysis on neuroimaging research of trigeminal neuropathic pain and temporomandibular joint disorders. PLoS One 9(4):e94300

25. Ayoub $L$, Seminowicz DA, Moayedi M (2018) A meta-analytic study of experimental and chronic orofacial pain excluding headache disorders. Neuroimage Clin 20:901-912

26. Moayedi M, Hodaie M (2019) Trigeminal nerve and white matter brain abnormalities in chronic orofacial pain disorders. Pain Rep 4(4):e755

27. Moher D, Shamseer $L$, Clarke $M$ et al (2015) Preferred reporting items for systematic review and meta-analysis protocols (PRISMA-P) 2015 statement. Syst Rev 4:1

28. Dworkin SF, LeResche L (1992) Research diagnostic criteria for temporomandibular disorders: review, criteria, examinations and specifications, critique. J Craniomandib Disord 6(4):301-355

29. Eric S, Richard O, Edmond T et al (2014) Diagnostic criteria for Temporomandibular disorders (DC/TMD) for clinical and research applications: recommendations of the international RDC/TMD consortium network* and Orofacial pain special interest groupt. J Oral Facial Pain Headache 28(1):6

30. von Elm E, Altman DG, Egger M et al (2007) The strengthening the reporting of observational studies in epidemiology (STROBE) statement: guidelines for reporting observational studies. Ann Intern Med 147(8):573-577

31. Younger JW, Shen YF, Goddard G et al (2010) Chronic myofascial temporomandibular pain is associated with neural abnormalities in the trigeminal and limbic systems. Pain 149(2):222-228

32. Gerstner G, Ichesco E, Quintero A et al (2011) Changes in regional gray and white matter volume in patients with myofascial-type temporomandibular disorders: a voxel-based morphometry study. J Orofac Pain 25(2):99-106

33. Melzack R (1987) The short-form McGill pain questionnaire. Pain 30(2):191-197

34. Spielberger CD. Measure anxiety, anger, depression, and curiosity in one inventory. Mind Garden website http://mindgarden.com/products/stpi.htm

35. Gustin SM, Peck CC, Wilcox SL et al (2011) Different pain, different brain: thalamic anatomy in neuropathic and non-neuropathic chronic pain syndromes. J Neurosci 31(16):5956-5964

36. Melzack R (1975) The McGill pain questionnaire: major properties and scoring methods. Pain 1(3):277-299

37. Beck AT, Ward CH, Mendelson M et al (1961) An inventory for measuring depression. Arch Gen Psychiatry 4:561-571

38. Spielberger CD, Gorsuch RL, Lushene RE (1970) Manual for the state-trait anxiety inventory. Consulting Psychologists Press, Polo Alto

39. Moayedi M, Weissman-Fogel I, Crawley AP et al (2011) Contribution of chronic pain and neuroticism to abnormal forebrain gray matter in patients with temporomandibular disorder. Neuroimage 55(1):277-286

40. Costa PT, McCrae RR (1992) Revised NEO personality (NEO-PI-R) and NEO five factor inventory (NEO-FFI) professional manual. Psychology Assessment Resources, Odessa, Florida

41. Moayedi M, Weissman-Fogel I, Salomons TV et al (2012) Abnormal gray matter aging in chronic pain patients. Brain Res 1456:82-93

42. Moayedi M, Weissman-Fogel I, Salomons TV et al (2012) White matter brain and trigeminal nerve abnormalities in temporomandibular disorder. Pain 153(7):1467-1477 
43. Salomons TV, Moayedi M, Weissman-Fogel I et al (2012) Perceived helplessness is associated with individual differences in the central motor output system. Eur J Neurosci 35(9):1481-1487

44. Sullivan MJL, Bishop SR, Pivik J (1995) The pain Catastrophizing scale: development and validation. Psychol Assess 7(4):524-532

45. Wilcox SL, Gustin SM, Eykman EN et al (2013) Trigeminal nerve anatomy in neuropathic and non-neuropathic orofacial pain patients. J Pain 14(8):865-872

46. Kucyi A, Moayedi M, Weissman-Fogel I et al (2014) Enhanced medial prefrontal-default mode network functional connectivity in chronic pain and its association with pain rumination. J Neurosci 34(11):3969-3975

47. He SS, Li F, Song F et al (2014) Spontaneous neural activity alterations in temporomandibular disorders: a cross-sectional and longitudinal resting-state functional magnetic resonance imaging study. Neuroscience 278:1-10

48. He S, Li F, Gu T et al (2018) Reduced corticostriatal functional connectivity in temporomandibular disorders. Hum Brain Mapp 39(6):2563-2572

49. Zhang J, Li X, Jin Z et al (2018) Spontaneous brain activity and connectivity in female patients with temporomandibular joint synovitis pain: a pilot functional magnetic resonance imaging study. Oral Surg Oral Med Oral Pathol Oral Radiol 126(4):363-374

50. Gustin SM, Peck CC, Cheney LB et al (2012) Pain and plasticity: is chronic pain always associated with somatosensory cortex activity and reorganization? J Neurosci 32(43):14874-14884

51. Ichesco E, Quintero A, Clauw DJ et al (2012) Altered functional connectivity between the insula and the cingulate cortex in patients with temporomandibular disorder: a pilot study. Headache 52(3):441-454

52. Nebel MB, Folger $\mathrm{S}$, Tommerdahl $\mathrm{M}$ et al (2010) Temporomandibular disorder modifies cortical response to tactile stimulation. J Pain 11(11):1083-1094

53. Zhao YP, Ma XC, Jin Z et al (2011) Cerebral activation during unilatera clenching in patients with temporomandibular joint synovitis and biting pain: an functional magnetic resonance imaging study. Chin Med J 124(14): 2136-2143

54. Weissman-Fogel I, Moayedi M, Tenenbaum HC et al (2011) Abnormal cortical activity in patients with temporomandibular disorder evoked by cognitive and emotional tasks. Pain 152(2):384-396

55. Roy A, Wang WE, Ho RLM et al (2018) Functional brain activity during motor control and pain processing in chronic jaw pain. Pain 159(12):2547-2564

56. Harper DE, Shah Y, Ichesco E et al (2016) Multivariate classification of painevoked brain activity in temporomandibular disorder. Pain Rep 1:3

57. He SS, Li F, Gu T et al (2016) Altered neural activation pattern during teeth clenching in temporomandibular disorders. Oral Dis 22(5):406-414

58. Gerstner GE, Gracely RH, Deebajah A et al (2012) Posterior insular molecular changes in myofascial pain. J Dent Res 91(5):485-490

59. Harfeldt K, Alexander L, Lam J et al (2018) Spectroscopic differences in posterior insula in patients with chronic temporomandibular pain. Scand Pain 18(3):351-361

60. Lickteig R, Lotze M, Kordass B (2013) Successful therapy for temporomandibular pain alters anterior insula and cerebellar representations of occlusion. Cephalalgia 33(15):1248-1257

61. DaSilva AF, Becerra L, Makris $N$ et al (2002) Somatotopic activation in the human trigeminal pain pathway. J Neurosci 22(18):8183-8192

62. Henderson LA, Peck CC, Petersen ET et al (2013) Chronic pain: lost inhibition? J Neurosci 33(17):7574-7582

63. Moulton EA, Pendse G, Morris S et al (2007) Capsaicin-induced thermal hyperalgesia and sensitization in the human trigeminal nociceptive pathway: an fMRI study. Neuroimage 35(4):1586-1600

64. Tracey I (2005) Nociceptive processing in the human brain. Curr Opin Neurobiol 15(4):478-487

65. Taylor KS, Seminowicz DA, Davis KD (2009) Two systems of resting state connectivity between the insula and cingulate cortex. Hum Brain Mapp 30(9):2731-2745

66. Mullins PG, Rowland LM, Jung RE et al (2005) A novel technique to study the brain's response to pain: proton magnetic resonance spectroscopy. Neuroimage 26(2):642-646

67. Yang CZ, Zhao R, Dong $Y$ et al (2008) Astrocyte and neuron intone through glutamate. Neurochem Res 33(12):2480-2486

68. Buckner RL, Andrews-Hanna JR, Schacter DL (2008) The brain's default network: anatomy, function, and relevance to disease. Ann N Y Acad Sci 1124:1-38
69. Chen Q, Heinricher MM (2019) Descending control mechanisms and chronic pain. Curr Rheumatol Rep 21(5):13

70. Hadjipavlou G, Dunckley P, Behrens TE et al (2006) Determining anatomical connectivities between cortical and brainstem pain processing regions in humans: a diffusion tensor imaging study in healthy controls. Pain 123(1-2): 169-178

71. Tenenbaum HC, Mock D, Gordon AS et al (2001) Sensory and affective components of orofacial pain: is it all in your brain? Crit Rev Oral Biol Med 12(6):455-468

72. Goldberg MB, Mock D, Ichise M et al (1996) Neuropsychologic deficits and clinical features of posttraumatic temporomandibular disorders. J Orofac Pain 10(2):126-140

73. Wright Cl, Williams D, Feczko E et al (2006) Neuroanatomical correlates of extraversion and neuroticism. Cereb Cortex 16(12):1809-1819

74. Weffort SY, de Fantini SM (2010) Condylar displacement between centric relation and maximum intercuspation in symptomatic and asymptomatic individuals. Angle Orthod 80(5):835-842

75. Barrera-Mora JM, Espinar Escalona E, Abalos Labruzzi C et al (2012) The relationship between malocclusion, benign joint hypermobility syndrome, condylar position and TMD symptoms. Cranio 30(2):121-130

76. Crawford SD (1999) Condylar axis position, as determined by the occlusion and measured by the CPI instrument, and signs and symptoms of temporomandibular dysfunction. Angle Orthod 69(2):103-115 discussion 115-106

77. Nachev P, Kennard C, Husain M (2008) Functional role of the supplementary and pre-supplementary motor areas. Nat Rev Neurosci 9(11):856-869

78. Helkimo M (1974) Studies on function and dysfunction of the masticatory system. II Index for anamnestic and clinical dysfunction and occlusal state. Sven Tandlak Tidskr 67(2):101-121

79. Schmidt-Wilcke T, Ganssbauer S, Neuner T et al (2008) Subtle grey matter changes between migraine patients and healthy controls. Cephalalgia 28(1):1-4

80. Hollins M, Sigurdsson A (1998) Vibrotactile amplitude and frequency discrimination in temporomandibular disorders. Pain 75(1):59-67

81. Aggleton JP (1993) The contribution of the amygdala to normal and abnormal emotional states. Trends Neurosci 16(8):328-333

82. Pessoa L (2008) On the relationship between emotion and cognition. Nat Rev Neurosci 9(2):148-158

83. Von Korff M, Ormel J, Keefe FJ et al (1992) Grading the severity of chronic pain. Pain 50(2):133-149

84. Lickteig R, Lotze M, Lucas C et al (2012) Changes in cortical activation in craniomandibular disorders during splint therapy - a single subject fMRI study. Ann Anat 194(2):212-215

85. Shankland WE (2001) The trigeminal nerve. Part II: the ophthalmic division. Cranio 19(1):8-12

86. Dougherty RF, Ben-Shachar M, Deutsch GK et al (2007) Temporal-callosal pathway diffusivity predicts phonological skills in children. Proc Natl Acad Sci U S A 104(20):8556-8561

87. May A, Gaser C (2006) Magnetic resonance-based morphometry: a window into structural plasticity of the brain. Curr Opin Neurol 19(4):407-411

88. Schwartz ED, Cooper ET, Fan Y et al (2005) MRI diffusion coefficients in spinal cord correlate with axon morphometry. Neuroreport 16(1):73-76

89. Guo W, Wang H, Zou S et al (2010) Long lasting pain hypersensitivity following ligation of the tendon of the masseter muscle in rats: a model of myogenic orofacial pain. Mol Pain 6:40

90. Gujar SK, Maheshwari S, Bjorkman-Burtscher I et al (2005) Magnetic resonance spectroscopy. J Neuroophthalmol 25(3):217-226

91. Ji RR, Xu ZZ, Gao YJ (2014) Emerging targets in neuroinflammation-driven chronic pain. Nat Rev Drug Discov 13(7):533-548

92. Baliki MN, Geha PY, Apkarian AV et al (2008) Beyond feeling: chronic pain hurts the brain, disrupting the default-mode network dynamics. J Neurosci 28(6):1398-1403

93. Vogt BA (2005) Pain and emotion interactions in subregions of the cingulate gyrus. Nat Rev Neurosci 6(7):533-544

94. Ong WY, Stohler CS, Herr DR (2019) Role of the prefrontal cortex in pain processing. Mol Neurobiol 56(2):1137-1166

95. Andrews-Hanna JR, Reidler JS, Sepulcre J et al (2010) Functional-anatomic fractionation of the brain's default network. Neuron 65(4):550-562

96. Kucyi A, Salomons TV, Davis KD (2013) Mind wandering away from pain dynamically engages antinociceptive and default mode brain networks. Proc Natl Acad Sci U S A 110(46):18692-18697 
97. Treede RD, Apkarian AV, Bromm B et al (2000) Cortical representation of pain: functional characterization of nociceptive areas near the lateral sulcus. Pain 87(2):113-119

98. DaSilva AF, Granziera C, Tuch DS et al (2007) Interictal alterations of the trigeminal somatosensory pathway and periaqueductal gray matter in migraine. Neuroreport 18(4):301-305

99. Seminowicz DA, Labus JS, Bueller JA et al (2010) Regional gray matter density changes in brains of patients with irritable bowel syndrome. Gastroenterology 139(1):48-57.e42

100. Stamford JA (1995) Descending control of pain. Br J Anaesth 75(2):217-227

101. Crombez G, Van Ryckeghem DM, Eccleston C et al (2013) Attentional bias to pain-related information: a meta-analysis. Pain 154(4):497-510

102. Seminowicz DA, Davis KD (2007) Interactions of pain intensity and cognitive load: the brain stays on task. Cereb Cortex 17(6):1412-1422

103. Seminowicz DA, Davis KD (2007) Pain enhances functional connectivity of a brain network evoked by performance of a cognitive task. J Neurophysiol 97(5):3651-3659

104. Romero YR, Straube T, Nitsch A et al (2013) Interaction between stimulus intensity and perceptual load in the attentional control of pain. Pain 154(1): $135-140$

105. Etkin A, Egner T, Peraza DM et al (2006) Resolving emotional conflict: a role for the rostral anterior cingulate cortex in modulating activity in the amygdala. Neuron 51(6):871-882

106. Whalen PJ, Bush G, Shin LM et al (2006) The emotional counting Stroop: a task for assessing emotional interference during brain imaging. Nat Protoc 1(1):293-296

107. Downar J, Mikulis DJ, Davis KD (2003) Neural correlates of the prolonged salience of painful stimulation. Neuroimage 20(3):1540-1551

108. Seeley WW, Menon V, Schatzberg AF et al (2007) Dissociable intrinsic connectivity networks for salience processing and executive control. J Neurosci 27(9):2349-2356

109. Kotov R, Gamez W, Schmidt F et al (2010) Linking "big" personality traits to anxiety, depressive, and substance use disorders: a meta-analysis. Psychol Bull 136(5):768-821

110. Hakulinen C, Elovainio M, Pulkki-Raback L et al (2015) Personality and depressive symptoms: individual participant meta-analysis of 10 cohort studies. Depress Anxiety 32(7):461-470

111. Harkins SW, Price DD, Braith J (1989) Effects of extraversion and neuroticism on experimental pain, clinical pain, and illness behavior. Pain 36(2):209-218

112. Goubert L, Crombez G, Van Damme S (2004) The role of neuroticism, pain catastrophizing and pain-related fear in vigilance to pain: a structural equations approach. Pain 107(3):234-241

113. Farina S, Tinazzi M, Le Pera D et al (2003) Pain-related modulation of the human motor cortex. Neurol Res 25(2):130-142

114. Boudreau S, Romaniello A, Wang K et al (2007) The effects of intra-oral pain on motor cortex neuroplasticity associated with short-term novel tongueprotrusion training in humans. Pain 132(1-2):169-178

115. Maier SF, Seligman ME (1976) Learned helplessness: theory and evidence. J Exp Psychol Gen 105(1):3-46

116. Salomons TV, Johnstone T, Backonja MM et al (2004) Perceived controllability modulates the neural response to pain. J Neurosci 24(32): 7199-7203

117. Nelson AB, Kreitzer AC (2014) Reassessing models of basal ganglia function and dysfunction. Annu Rev Neurosci 37:117-135

118. Provost JS, Hanganu A, Monchi O (2015) Neuroimaging studies of the striatum in cognition part I: healthy individuals. Front Syst Neurosci 9:140

119. Chudler EH (1998) Response properties of neurons in the caudate-putamen and globus pallidus to noxious and non-noxious thermal stimulation in anesthetized rats. Brain Res 812(1-2):283-288

120. Hollins M, Harper D, Gallagher S et al (2009) Perceived intensity and unpleasantness of cutaneous and auditory stimuli: an evaluation of the generalized hypervigilance hypothesis. Pain 141(3):215-221

121. Gil-Martinez A, Paris-Alemany A, Lopez-de-Uralde-Villanueva I et al (2018) Management of pain in patients with temporomandibular disorder (TMD): challenges and solutions. J Pain Res 11:571-587

122. Armijo-Olivo S, Pitance $L$, Singh $V$ et al (2016) Effectiveness of manual therapy and therapeutic exercise for Temporomandibular disorders: systematic review and meta-analysis. Phys Ther 96(1):9-25

123. Fricton J, Look JO, Wright E et al (2010) Systematic review and metaanalysis of randomized controlled trials evaluating intraoral orthopedic appliances for temporomandibular disorders. J Orofac Pain 24(3):237-254
124. Harada T, Ichiki R, Tsukiyama Y et al (2006) The effect of oral splint devices on sleep bruxism: a 6-week observation with an ambulatory electromyographic recording device. J Oral Rehabil 33(7):482-488

125. Nascimento LL, Amorim CF, Giannasi LC et al (2008) Occlusal splint for sleep bruxism: an electromyographic associated to Helkimo index evaluation. Sleep Breath 12(3):275-280

126. Dao T, Lavigne GJ (1998) Oral splints: the crutches for temporomandibular disorders and bruxism? Crit Rev Oral Biol Med 9(3):345-361

127. Fricton J (2007) Myogenous temporomandibular disorders: diagnostic and management considerations. Dent Clin N Am 51(1):61-83 vi

128. Carlsson GE (1999) Epidemiology and treatment need for temporomandibular disorders. J Orofac Pain 13(4):232-237

129. Huber MA, Hall EH (1990) A comparison of the signs of temporomandibular joint dysfunction and occlusal discrepancies in a symptom-free population of men and women. Oral Surg Oral Med Oral Pathol 70(2):180-183

130. Schiffman E, Fricton JR (1989) Epidemiology of TMJ and craniofacial pains: an unrecognized societal problem. In: Fricton JR, Kroening RJ, Hathaway KM (eds) TMJ and craniofacial pain: diagnosis and management. Ishiyaku EuroAmerica, St. Louis, pp 1-10

131. Stegenga $B$, de Bont $L G$, de Leeuw $R$ et al (1993) Assessment of mandibular function impairment associated with temporomandibular joint osteoarthrosis and internal derangement. J Orofac Pain 7(2):183-195

132. Gonzalez YM, Schiffman E, Gordon SM et al (2011) Development of a brief and effective temporomandibular disorder pain screening questionnaire: reliability and validity. J Am Dent Assoc 142(10):1183-1191

\section{Publisher's Note}

Springer Nature remains neutral with regard to jurisdictional claims in published maps and institutional affiliations.
Ready to submit your research? Choose BMC and benefit from:

- fast, convenient online submission

- thorough peer review by experienced researchers in your field

- rapid publication on acceptance

- support for research data, including large and complex data types

- gold Open Access which fosters wider collaboration and increased citations

- maximum visibility for your research: over $100 \mathrm{M}$ website views per year

At BMC, research is always in progress.

Learn more biomedcentral.com/submissions 